\title{
Carvedilol vs. metoprolol: A comparison of effects on endothelial function and oxidative stress in response to acute hyperglycemia in patients with type 2 diabetes and hypertension
}

\author{
Kathleen Colleran", Lina Aguirre, Mark R. Burge \\ Department of Internal Medicine, University of New Mexico Health Sciences Center, Albuquerque, USA; \\ ${ }^{\#}$ Corresponding Author: KColleran@,salud.unm.edu
}

Received 16 November 2011; revised 15 December 2011; accepted 31 December 2011

\begin{abstract}
Introduction: The GEMINI trial compared the effects of treatment with metoprolol versus carvedilol in patients with type 2 diabetes. Carvedilol demonstrated a more favorable effect on factors associated with the metabolic syndrome than metoprolol. We hypothesize that carvedilol will have additional beneficial effects on markers of inflammation, oxidative stress, and endothelial function than metoprolol. Methods: Twenty subjects were randomized to either carvedilol or metoprolol. Study procedures including assesment of metabolic parameters and endothelial function, while fasting and after a $75 \mathrm{~g}$ oral glucose tolerance were conducted at baseline and following 5 months of treatment. Results: Following 5 months of treatment, PAI-1 increased significantly from baseline in the metoprolol group. There were no changes in PAI-1 in the carvedilol group. While not reaching statistical significance, there was a trend toward worsening insulin resistance with metoprolol treatment compared to carvedilol treatment. Flow mediated vasodilation increased in both groups following the 2-hr OGGT during the baseline study. After five months of treatment, there was a nonsignificant increase in flow-mediated vasodilation under both fasting and post OGTT conditions in the carvedilol group compared to baseline. Conversely, there was no change in fasting flow mediated vasodilation in the metoprolol group. Additionally, metoprolol treatment blunted the increase in flow mediated vasodilation fol-

*Funding sources: This projects was supported by the University of New Mexico Clinical and Translational Science Center, CTSA 1ULRR031977.

Glaxo Smith Kline funded this investigator initiated project.
\end{abstract}

lowing OGGT compared to baseline $(p<0.05)$. Conclusion: Treatment with metoprolol was associated with adverse metabolic effects including increases in PAI-1 and trends toward worsening insulin resistance and endothelial function compared to treatment with carvedilol.

Keywords: Diabetes; Hypertension; Endothelial Dysfunction; Carvedilol; Metoprolol; Insulin Resistance

\section{INTRODUCTION}

Over $75 \%$ of patients with diabetes die from cardiovascular disease (CVD) related complications. Prevention of CVD risk factors including hyperglycemia and hypertension has been demonstrated to reduce CVD events in diabetics $[1,2]$. Beta blockers have been a cornerstone in the treatment of hypertension and secondary prevention of CVD and congestive heart failure [1]. However, concerns about masking hypoglycemic symptoms and worsening glycemic control have prevented wide use of beta blockers in diabetics $[1,3]$.

Worsening hyperglycemia is of particular concern, as numerous studies have shown that worsening insulin resistance increases CVD risk factors including inflammation, oxidative stress and endothelial function [4-8]. The recently published GEMINI trial-compared the selective beta-1 receptor antagonist, metoprolol, against the non-selective $\beta$ - and $\alpha 1$-receptor antagonist, carvedilol, in patients with type 2 diabetes. Both drugs were effective in reducing blood pressure and were well tolerated [9]. Interestingly, carvedilol demonstrated a more favorable effect on factors associated with the metabolic syndrome compared to metoprolol. An investigator participating in the GEMINI trial completed an additional investigator initiated sub study to measure the effects of 
carvedilol and metoprolol on endothelial function. The investigators demonstrated that carvedilol significantly improved endothelial function compared to metoprolol [10]. To further test the hypothesis that carvedilol has unique beneficial effects on endothelial function, inflammation, and oxidative stress compared to metoprolol in patients with type 2 diabetes, we performed the following study.

\section{METHODS}

\subsection{Patient Population}

This study was conducted as a sub study to the GEMINI study [9]. Inclusion and exclusion criteria have been described in detail in the GEMINI publication. Briefly, twenty subjects with type 2 diabetes and uncontrolled hypertension despite current treatment with an Angiotensin Converting Enzyme Inhibitor (ACE) or an Angiotensin Receptor Blocker (ARB) were enrolled and randomized to receive either carvedilol or metoprolol. Verbal and written consent was obtained from each subject. The study was approved by Human Research and Review Committee (HRRC), the local Institutional Review Board at the University of New Mexico Health Sciences Center. All procedures were conducted in accordance with the HRRC and the Health Insurance Portability and Accountability Act policies. Clinical Trials Number: NCT00642434.

\subsection{Study Design}

The study design was identical to that of the parent study, and is described in detail elsewhere [9]. Subjects continued their ACE/ARB throughout the study. They were washed out of all other antihypertensive agents. Study medication was titrated to maximum dose. If blood pressure was not at the study protocol designated target of $<130 / 80 \mathrm{mmHg}$, hydrochlorothiazide $25 \mathrm{mg}$ was added. Amlodipine was added if blood pressure was still not at goal. Once goal blood pressure was reached, subjects were maintained on study treatment for five months.

\subsection{Study Protocols and Procedures}

All subjects underwent a baseline study, following a two week washout of all anti-hypertensives except for ACE/ARBs. All study procedures were repeated after five months of study intervention.

Subjects were admitted to the inpatient General Clinical Research Center in the evening. They received a standard ADA meal, and then began an observed 12-hour fast. The following morning, subjects underwent a blood test followed by a study of vascular endothelial function (see Section 2.5 Endothelial Function). Subjects then underwent a 75 gram oral glucose tolerance. Two hours later blood tests were repeated and endothelial function was retested.

\subsection{Laboratory Analyses}

Samples were taken to measure lipids, glycemic control (adiponectin, HbA1c). Glucose and insulin were used to calculate Homeostasis Model Assessment: Insulin resistance (HOMA-IR) [11]. Inflammation was assessed by highly sensitive C-reactive protein (hsCRP) and interleukin six (IL-6). Coagulation/thromobosis parameters were assessed by Plasminogen Activator Inhibitor one (PAI-1), fibrinogen, and homocysteine (HCY).

HCY was processed in pre-iced, 4-mL EDTA tubes, immediately placed on ice, and centrifuged. Concentrations were determined by Immulite Chemiluminescence, Diagnostic Products Corporation, (reference range, 5 $15 \mu \mathrm{mol} / \mathrm{L}$, GCRC labs, Albuquerque, NM). PAI-1 activity was measured using a Chromolize PAI-1 kit by biopool, Kit catalog number: 1106, (Method: A "Sandwich type" enzyme-linked immunosorbent assay), (reference range 2 - $50 \mathrm{IU} / \mathrm{ml}$ GCRC labs, Albuquerque, NM). HsCRP concentration was determined by immunometric assay, IMMULITE-high sensitivity CRP, (reference range: undetectable-1.1 mg/dl, GCRC labs, Albuquerque, $\mathrm{NM}$ ). Glucose was processed in $8.5 \mathrm{~mL} \mathrm{SST}$, centrifuged and measured on the ACE instrument, (Hexokinase method) manufactured by Alfa Wassermann, Inc. West Caldwell, NJ, (reference range 79.3 - $129.2 \mathrm{mg} / \mathrm{dL}$, GCRC core Lab Albuquerque, NM). Total cholesterol, LDL, HDL and triglycerides were measured by spectrophotometry, (reference range, $<200 \mathrm{mg} / \mathrm{dL},<100 \mathrm{mg} / \mathrm{dL}$, $>40 \mathrm{mg} / \mathrm{dL}$, and $<150 \mathrm{mg} / \mathrm{dL}$, respectively, Tricore Reference Labs, Albuquerque, $\mathrm{NM}$ ). $\mathrm{HbA}_{1 \mathrm{c}}$ was measured by inhibition of latex agglutination, (DCA 2000 Hemoglobin A1c reagent kit, Bayer Corporation, USA), (reference range, 4.4\% - 5.8\%, GCRC labs, Albuquerque, NM).

Adiponectin was measured by ELISA, (reference range, $2-40 \mu \mathrm{g} / \mathrm{mL}$, GCRC labs, Albuquerque, NM). High Sensitivity IL-6 was measured by ELISA, (reference range, condition dependent, calibration range, 0.156 - 10 pg/mL, GCRC labs, Albuquerque, NM). Insulin was measured by Immulite (Chemiluminescence), (reference range, 2 - $28.4 \mu \mathrm{IU} / \mathrm{mL}$, GCRC labs, Albuquerque, NM).

\subsection{Endothelial Function}

Vascular endothelial function was assessed by measuring flow mediated dilation (FMD) of the brachial artery in response to hyperemia, using standard techniques [12]. Specifically, all studies were conducted in a temperature-controlled room. Subjects were placed comfortably in a supine resting position with the left arm immobilized for at least fifteen minutes prior to study 
procedures. A sphygmomanometer cuff was positioned above the antecubital fossa on the upper left arm. A baseline brachial artery diameter was measured using a 7.5 $\mathrm{MHz}$ linear array ultrasound. Diameter measurements were taken from one media-adventitia interface to the other. The brachial artery diameter was measured three times, and the average was recorded. Following baseline measurement, endothelial function was assessed following ischemic hyperemia. The cuff was inflated to 200 $\mathrm{mmHg}$ or $60 \mathrm{mmHg}$ above systolic blood pressure for five minutes. The brachial artery diameter was remeasured at one minute post-deflation. After five minutes of recuperation, the subjects took $0.4 \mathrm{mg}$ of sublingual nitroglycerin, and the brachial artery diameter measurements were repeated to evaluate endothelial independent dilation. Percent increase in brachial artery diameter from baseline following both postischemic hyperemia and nitroglycerine administration was calculated and represents the FMD endpoint for endothelial dependent and endothelial independent function respectively.

\subsection{Statistical Analysis}

Data were analyzed by SAS/STAT® Version 8.0 (SAS Institute Inc., Cary, NC). The data were analyzed using student's t-tests and repeated measures ANOVA. Descriptive data were reported as a mean \pm standard deviation. A $p<0.05$ was considered significant.

\section{RESULTS}

Twenty subjects met enrollment criteria, completed all study visits, and were thus included in the final analysis. Nine subjects were randomized to metoprolol and eleven subjects were randomized to carvedilol. At baseline, there were no differences in parameters of glucose control, markers of inflammation, oxidative stress or endothelial function.

\section{Labs}

Following five months of treatment with study drug, there were no significant differences IL-6, hsCRP, or adiponectin (Table 1). Interestingly, PAI-1, an adipocytokine associated with thrombolysis, was significantly increased from baseline following five months of metoprolol treatment $(19.5 \pm 24 \mathrm{IU} / \mathrm{mL}$ versus $28.4 \pm 26$ $\mathrm{IU} / \mathrm{mL}$, respectively, $\mathrm{p}<0.05)$. There were no significant changes in PAI-1 in the group treated with carvedilol (20 $\pm 12.5 \mathrm{IU} / \mathrm{mL}$ versus $24.5 \pm 16 \mathrm{IU} / \mathrm{mL}$, respectively, $\mathrm{p}=$ ns) (Table 1).

Additionally, while not reaching statistical significance, important trends suggest that that metoprolol and carvedilol may differ in their effects on insulin sensitivity and glycemic control. Specifically, subjects treated with metoprolol had a non-significant increase in $\mathrm{HbA1c}$ compared to baseline after five months of treatment (6.8 $\pm 0.8 \%$ versus $7.1 \pm 1.1 \%$ respectively) and HOMA IR (94 \pm 58 versus $130 \pm 84$ respectively) compared to those treated with carvediolol $(\mathrm{HbA} 1 \mathrm{c}(6.6 \pm 0.6 \%$ versus $6.5 \pm$ $0.9 \%$ respectively), and HOMA-IR ( $86 \pm 90$ versus 93.7 \pm 65 respectively) (Table 2 ).

Vascular data are shown in Figure 1. At baseline, there were no significant differences in resting brachial artery diameter or FMD between the metoprolol and carvedilol groups. Acute hyperglycemia led to an increase in resting brachial artery diameter in both groups at baseline. Following five months of treatment, there were no differences in resting brachial artery diameter compared to baseline in either group. However, the group receiving carvedilol demonstrated a non-significant trend toward increasing FMD compared to baseline. These changes were seen under both fasting and after acute hyperglycemia. Conversely, FMD in the fasting state was unchanged in the metoprolol group following five months of treatment. Interestingly, metoprolol treatment blunted acute hyperglycemia induced increases in FMD $(\mathrm{p}<0.05)$.

Study drug was well tolerated in both groups, and neither group experienced any adverse effects throughout the course of the study.

Table 1. Changes in markers of inflammation following treatment with carvedilol versus metoprolol.

\begin{tabular}{|c|c|c|c|c|c|c|}
\hline & \multicolumn{3}{|c|}{ Carvedilol } & \multicolumn{3}{|c|}{ Metoprolol } \\
\hline & Baseline & $\begin{array}{l}\text { Post-Treatment } \\
\text { (Month 5) }\end{array}$ & Delta & Baseline & $\begin{array}{l}\text { Post-Treatment } \\
\text { (Month 5) }\end{array}$ & Delta \\
\hline $\begin{array}{c}\text { PAI-1 } \\
(2-50 \mathrm{IU} / \mathrm{ml})\end{array}$ & $20 \pm 12.5$ & $24.5 \pm 16$ & 4.5 & $19.5 \pm 24$ & $28.4 \pm 26^{*}$ & $8.9^{*}$ \\
\hline $\begin{array}{c}\text { Adiponectin } \\
(2-40 \mu \mathrm{g} / \mathrm{mL})\end{array}$ & $15.7 \pm 14$ & $19.4 \pm 20$ & 3.7 & $14.1 \pm 13$ & $11 \pm 7.3$ & -3.3 \\
\hline $\begin{array}{c}\mathrm{Il}-6 \\
(0.156-10 \mathrm{pg} / \mathrm{mL})\end{array}$ & $2.1 \pm 0.1$ & $2.6 \pm 2$ & 0.5 & $2.3 \pm 0.7$ & $2.2 \pm 1$ & 0.1 \\
\hline $\begin{array}{c}\text { hsCRP } \\
(<0.1-1.1 \mathrm{mg} / \mathrm{dl})\end{array}$ & $0.4 \pm 0.4$ & $0.5 \pm 0.6$ & 0.1 & $0.6 \pm 0.4$ & $0.6 \pm 0.5$ & 0.0 \\
\hline
\end{tabular}

*Significant value $(\mathrm{p}<0.05)$. 
Table 2. Changes in markers of insulin resistance following treatment with carvedilol versus metoprolol.

\begin{tabular}{|c|c|c|c|c|c|c|c|c|}
\hline & \multicolumn{4}{|c|}{ Carvedilol } & \multicolumn{4}{|c|}{ Metoprolol } \\
\hline & \multicolumn{2}{|c|}{ Baseline } & \multicolumn{2}{|c|}{ Post-Treatment (Month 5) } & \multicolumn{2}{|c|}{ Baseline } & \multicolumn{2}{|c|}{ Post-Treatment (Month 5) } \\
\hline & Fasting & Post OGTT & Fasting & Post OGTT & Fasting & Post OGTT & Fasting & Post OGTT \\
\hline $\begin{array}{c}\text { Glucose } \\
(79-129 \mathrm{mg} / \mathrm{dL})\end{array}$ & $122 \pm 22$ & $263 \pm 85$ & $120 \pm 24$ & $259 \pm 67$ & $131 \pm 30$ & $294 \pm 64$ & $147 \pm 56$ & $297 \pm 85$ \\
\hline $\begin{array}{c}\text { Insulin } \\
(2-28 \mu \mathrm{IU} / \mathrm{mL})\end{array}$ & $17.9 \pm 14$ & $60.4 \pm 26$ & $19.7 \pm 16$ & $79.4 \pm 67$ & $17.4 \pm 51$ & $50.8 \pm 32$ & $23.2 \pm 11$ & $76.3 \pm 67$ \\
\hline HOMA IR & $86 \pm 90$ & N/A & $93.7 \pm 65$ & N/A & $94 \pm 58$ & N/A & $130 \pm 84$ & N/A \\
\hline $\begin{array}{c}\text { HbA1c } \\
(4.4 \%-5.8 \%)\end{array}$ & $6.6 \pm 0.6$ & N/A & $6.5 \pm 0.9$ & $\mathrm{~N} / \mathrm{A}$ & $6.8 \pm 0.8$ & N/A & $7.1 \pm 1.1$ & N/A \\
\hline
\end{tabular}

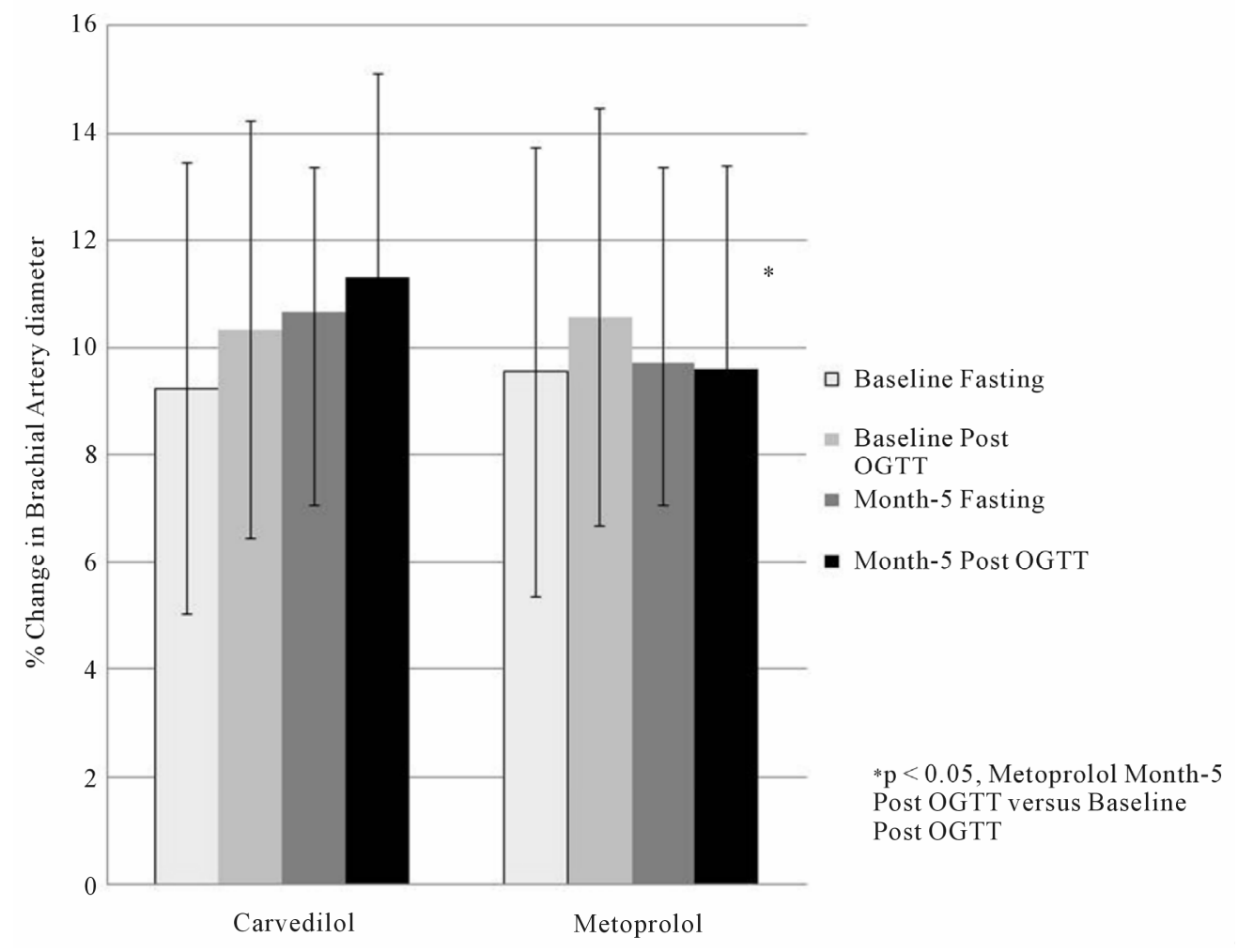

Figure 1. Change in brachial artery diameter following ischemic hyperemia before and after an OGTT at baseline and after 5 months of study drug.

\section{DISCUSSIONS}

In this study, we demonstrated that carvedilol and metoprolol are equally effective in reducing blood pressure and are well tolerated. However, carvedilol appears to have more beneficial metabolic and vascular effects compared to metoprolol. Specifically, subjects treated with carvedilol had lower PAI-1 activity compared to those treated with metoprolol. Additionally, two-hour post OGTT PAI-1 activity was lower in the group treated with carvediolol. PAI-1, is an antifibrinolytic protein. PAI-1 blocks the activation of plasminogen to plasmin thus preventing clot lysis. It is produced in numerous tissues including adipose tissues. Elevations in PAI-1 are associated with both CVD and insulin resistance [13, 14].

We did not find any significant differences in other novel CVD risk factors including inflammation and oxidative stress. However, numerous studies have demonstrated that carvedilol does indeed improve oxidative stress compared to other earlier or second generation beta blockers [15-18]. Our study may not have been powered to detect changes in inflammation and oxidative stress. 
We did, however, find a trend toward worsening insulin resistance and glycemic control in subjects treated with metoprolol. Similarly, others studies, including GEMINI, have demonstrated that that carvedilol has beneficial effects on insulin sensitivity compared to other beta blockers $[9,10]$.

\subsection{Ultra Sound}

Changes in FMD in the brachial artery did not differ by treatment group. However, there were trends suggesting that treatment with carvedilol may lead to improvement in FMD thus endothelial function compared to metoprolol. Previous studies have demonstrated similar results including another sub-study of the GEMINI Trial $[19,20]$.

Interestingly, acute hyperglycemia two hours following a 75 gram glucose challenge led to increased FMD compared to fasting under baseline conditions. Reasons for this are not clear but could include the osmotic effect of hyperglycemia initially increasing extracellular blood volume. However, in our study, increases in FMD following acute hyperglycemia were blunted following five months of treatment with metoprolol, but were unaffected by treatment with carvedilol. Further investigation is warranted.

\subsection{Limitations}

There were several limitations to our study. The study groups were unequal. Twelve subjects were randomized to the carvedilol treatment arm and eight to the metoprolol arm. Computer randomization should have prevented group assignment inequality. Unfortunately, there were two distinct randomization methods used for our cohort. The initial seven subjects were randomized as part of the multicenter study, GEMINI [9]. Following enrollment closure of GEMINI, we continued to enroll fifteen subjects for this sub study. Our research pharmacist created a new randomized program. The two different systems were not matched leading to unequal randomization to study groups. Additionally, two subjects in the second part of the study did not complete all study visits and were not included in the final analysis.

\section{CONCLUSION}

We conclude that beta blockers are efficacious in reducing blood pressure and are well tolerated in patients with type 2 diabetes. However, metoprolol has more adverse metabolic effects compared to carvedilol including increasing PAI-1 activity and trends toward worsening insulin resistance and impaired endothelial function.

\section{ACKNOWLEDGEMENTS}

The authors would like to thank Lisa Toelle for providing editing, formatting and other technical assistance.

\section{REFERENCES}

[1] Stamler, J., Vaccaro, O., Neaton, J.D. and Wentworth, D. (1993) For the multiple risk factor intervention trial research group. Diabetes, other risk factors, and the 12-year cardiovascular mortality for men screened in the multiple risk factor intervention trial. Diabetes Care, 16, 434-444. doi:10.2337/diacare.16.2.434

[2] Song, S.H. and Brown, P.M. (2004) Coronary heart disease risk assessment in diabetes mellitus: Comparison of UKPDS risk engine with framingham risk assessment function and its clinical implications. Diabetic Medicine, 21, 238-245. doi:10.1111/j.1464-5491.2004.01116.x

[3] Taylor, E.N., Hu, F.B. and Curhan, G.C. (2006) Antihypertensives medications and the risk of incident type 2 diabetes. Diabetes Care, 29, 1065-1070. doi: $10.2337 / \mathrm{dc} 05-2366$

[4] Kullo, I.J., Gau, G.T. and Tajik, A.J. (2000) Novel risk factors for atherosclerosis. Mayo Clinic Proceedings, 75, 369-380. doi: 10.4065/75.4.369

[5] Colagiuri, S., Cull, C.A. and Holman, R.R. (2002) Are lower fasting plasma glucose levels at diagnosis of type 2 diabetes associated with improved outcomes? UK prospective diabetes study 61. Diabetes Care, 25, 1410-1417. doi:10.2337/diacare.25.8.1410

[6] Kawamura, M., Heinecke, J.W. and Chait, A. (1994) Pathophysiological concentrations of glucose promote oxidative modification of low density lipoprotein by a superoxide-dependent pathway. The Journal of Clinical Investigation, 94, 771-778. doi:10.1172/JCI117396

[7] Cerriello, A. (1997) Acute hyperglycaemia and oxidative stress generation. Diabetic Medicine, 14, S45-S49. doi:10.1002/(SICI)1096-9136(199708)14:3+<S45::AIDDIA444>3.3.CO;2-I

[8] Cerriello, A., Bortolotti, N., Crescentini, A., Motz, E., Lizzio, S., Russo, A., Ezsol, Z., Tonutti, L. and Taboda, C. (1998) Antioxidant defenses are reduced during the oral glucose tolerance test in normal and non-insulin-dependent diabetic subjects. 1998. European Journal of Clinical Investigation, 28, 329-333. doi:10.1046/j.1365-2362.1998.00295.x

[9] Bakris, G.L., Fonseca, V., Katholi, R.E., McGill, J.B., Messerli, F.H., Phillips, R.A., Raskin, P., Wright, J.T., Oakes, R., Lukas, M.A., Anderson, K.M. and Bell, D.S. (2004) Metabolic effects of carvedilolvsmetoprolol in patients with type 2 diabetes mellitus and hypertension. A randomized controlled trial. Journal of the American Medical Association (JAMA), 292, 2227-2236. doi:10.1001/jama.292.18.2227

[10] Bank, A.J., Kelly, A.S., Thelen, A.M., Kaiser, D.R. and Gonzalez-Campoy, M.J. (2007) Effects of carvedilol versus metoprolol on endothelial function and oxidative stress in patients with type 2 diabetes. American Journal of Hypertension (AJH), 20, 777-783.

[11] Matthews, D.R., Hosker, J.P., Rudenski, A.S., Naylor, B.A., Treacher, D.F. and Turner, R.C. (1985) Homeostasis 
model assessment: Insulin resistance and beta-cell function from fasting plasma glucose and insulin concentrations in man. Diabetologia, 28, 412-419. doi:10.1007/BF00280883

[12] Perez, A., Leotta, D.F., Sullivan, J.H., et al. (2006) Flow mediated dilation of the brachial artery: An investigation of methods requiring further standardization. BMC Cardiovascular Disorders, 7, 11-19. doi:10.1186/1471-2261-7-11

[13] Vaughan, D.E. (1998) Plasminogen activator inhibitor-1: A common denominator in CVD. Journal Investigative Medicine, 46, 370-376.

[14] Huber, K., Christ, G., Wojta, J. and Gulba, D. (2001) Plasminogen activator inhibitor-Type 1 in CVD. Status Report 2001. Thrombosis Research, 103, S7-S19. doi:10.1016/S0049-3848(01)00293-6

[15] Feuerstein, G., Yue, T.L., Ma, X. and Ruffolo, R.R. (1998) Novel mechanisms in the treatment of heart failure: Inhibition of oxygen radicals and apoptosis by carvedilol. Progress in Cardiovascular Diseases, 412, 17-24. doi:10.1016/S0033-0620(98)80027-3

[16] Yue, T.L., McKenna, P.J., Gu, J.L., Cheng, H.Y., Ruffolo, R.R. and Feuerstein, G. (1994) Carvedilol, a new vasodi- lating $\mathrm{b}$ adrenoreceptor blocker antihypertensive drug, protects endothelial cells from damage initiated by xanthine-xanthine oxidase and neutrophils. Cardiovascular Research, 28, 400-406. doi:10.1093/cvr/28.3.400

[17] Lysko, P.G., Webb, C.L., GU, J.L., Ohlstein, E.H., Ruffolo, R.R. and Yue, T.L. (2000) A comparison of carvedilol and metoprolol antioxidant activities in vitro. Journal of Cardiovascular Pharmacology, 36, 277-281. doi:10.1097/00005344-200008000-00020

[18] Guiuliano, D., Acampora, R., Marfella, R., et al. (1997) Metabolic and cardiovascular effects of carvedilol and atenolol in non-insulin dependent diabetes mellitus and hypertension. Annals of Internal Medicine, 126, 955-959.

[19] Matsuda, Y., Akita, H., Terashima, M., Shiga, N., Kanazawa, K. and Yokoyama, M. (2000) Carvedilol improves endothelium-dependent dilatation in patients with coronary artery disease. American Heart Journal, 140, 753759.

[20] Bank, A., Kelly, A., Thelen, D. and Gonzalez-Campoy, M. (2007) Effectsof carvedilol versus metoprolol on endothelial function and oxidative stress in patients with type 2 diabetes mellitus. American Journal of Hypertension, 20, 777-783. doi:10.1016/j.amjhyper.2007.01.019 